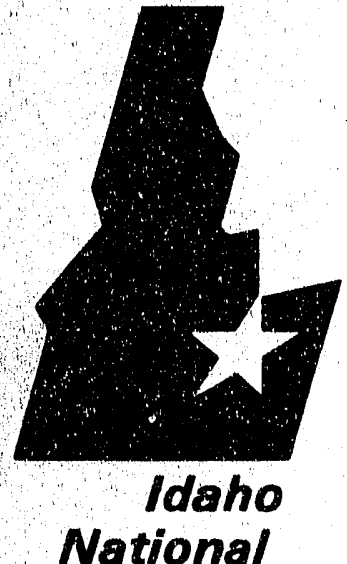

Engineering

Laboratory

Managed

by the U.S.

Department

of Energy
EGG-EP-9918

September 1991

\section{Alf $: 109$}

\title{
FEASIBILITY STUDIES FOR THE SONIC SENSOR SYSTEM FOR NONINVASIVE TEMPERATURE MEASUREMENT IN THE FOOD PROCESSING INDUSTRY
}

\author{
Laurence S. Beller \\ Charles R. Mikesell \\ Steven C. Taylor \\ David M. Tow
}

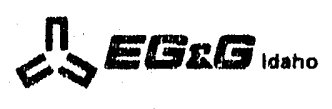

Work performed under DOE Contract No. DE-AC07-751D01570 
This document contains new concepts or the author(s) interpretation of new calculations and/or measurements; accordingly, EG\&G Idaho, Inc. is required by the United States Government to include the following disclaimer:

\section{DISCLAIMER}

This report was prepared as an accourt of work sponsored by an agency of the United States Government. Neither the United States Government nor any agency thereof, nor any of their employees, makes any warranty, express or implied, or assumes any legal liability or responsibility for the accuracy, completeness, or usefulness of any information, apparatus, product or process disclosed, or represents that its use would not infringe privately owned rights. References herein to any specific commercial product, process, or service by trade name, trademark, manufacturer, or otherwise, does not necessarily constitute or imply its endorsement, recommendation, or favoring by the United States Government or any agency thereof. The views and opinions of authors expressed herein do not necessarily state or reflect those of the United States Government or any agency thereof. 


\title{
FEASIBILITY STUDIES FOR THE SONIC SENSOR SYSTEM FOR NONINVASIVE TEMPERATURE MEASUREMENT IN THE FOOD PROCESSING INDUSTRY
}

\author{
Laurence S. Beller \\ Charles R. Mikesell \\ Steve C. Taylor \\ David M. Tow
}

Published September 1991

EG\&G Idaho, Inc.

Idaho Falls, Idaho $\mathbf{8 3 4 1 5}$

Prepared for the

U.S. Department of Energy

Assistant Secretary for Conservation and Renewable Energy

Under DOE Idaho Field Office

Contract DE-AC07-76ID01570 


\begin{abstract}
Feasibility studies for development of the Sonic Sensor System to measure temperature in the interior of particulates during food processing are described. The method involves tomography-based acquisition and interpretation of speed of sound data. The method is found to be feasible, and representative engineering problems and other technical issues are identified. A two-step approach to concept commercialization, involving replacement of the present puncture-and-thermometer method in conventional batch processing and development of a device to use in continuous aseptic processing, is proposed. A conservative estimate of immediate energy savings, just for food processing, is 0.01 quad/y. Potential follow-on applications are discussed.
\end{abstract}




\section{SUMMARY}

Current processing technology for particulate foods combines extremely conservative time/retort-temperature relationships with destructive product sampling for process control verification. Ongoing work at the Idaho National Engineering Laboratory under the direction of the U.S. Department of Energy is developing an ultrasound-based method to determine the particulate-interior temperature non-invasively. The use of this method has potential for substantial energy savings. Technical expertise with respect to current industrial food-processing technology and its problems has been obtained through cooperation with the National Food Processors Association (NFPA).

The method in its present form determines the speed of ultrasound in the two-phase (liquid plus particulate food) product. Underlying standards are the ultrasound-speed-versus-temperature relationships for both water and food materials. Data acquisition and interpretation are based on tomography to identify each phase separately, determine their temperatures from speed of sound versus temperature relationships of each phase separately, and ensure that the data are representative of the overall product.

Recently completed feasibility studies investigated the acoustic properties of seleited food materials, the consequent requirements of the tomographic process, and design approaches for the ultrasonic sensor. The studies show that the concept is technically feasible, and indicate some of the engineering problems and other technical issues to be resolved in design and fabrication of prototypes.

Noninvasive product temperature me asurement for current food-processing technology appears relatively straightforward, and could achieve energy savings of 0.01 quad/y if fully implemented on a national scale. Extension of the method to aseptic processing (subject to regulatory action) could result in substantially larger energy savings in addition to economic and product quality benefits.

A proposal for a cooperative study with the NFPA for development of a laboratory prototype based on this concept has been submitted to the Department of Energy. The scope of the proposal includes detailed determination of sonic properties of fond materials, system design and test, and pilot-plant testing. A second set if tasks will address design, fabrication, and laboratory testing of the sensing element of a device for aseptic processing. Activities beyond this phase directed toward development of a commercial prototype system and ultimate commercialization of the process, are discussed. 


\section{ACKNOWLEDGMENTS}

The authors gratefully acknowledge the valuable contributions of the following in the planning, conduct, and review of this feasibility study:

- Mr. H. Brown, Consultant--for assimilating, structuring, and editing the information and contents of this report.

- Mr. W. Akers, EG\&G Idaho, Inc.--for providing program line management

- Dr. L. Pedersen, NFPA--for functioning as the industrial consultant for this project 


\section{CONTENTS}

ABSTRACT ............................

SUMMARY ..................... iv

ACKNOWLEDGMENTS. .....................

1. INTRODUCTION AND OVERVIEW ......................... 1

1.1 The Need for a Sensor . . . . . . . . . . . . . . . 1

1.1.1 Conventional Methods . . . . . . . . . . . . . . 1

1.1 .2 The Aseptic Process . . . . . . . . . . . . . 2

1.2 The Sonic Sensor System Concept . . . . . . . . . . . . . . 4

1.2.1 Measurement of Temperature by the Speed of Sound . . . . 4

1.2 .2 The Tomographic Process . . . . . . . . . . 5

2. FEASIBILITY STUdIES ................... 13

2.1 Questions for the Feasibility Studies . . . . . . . . . 13

2.2 Experimental Measurement of Acoustic Properties of Food 16

2.2.1 Test Material . . . . . . . . . . . 16

2.2.2 Apparatus ................. 17

2.3 Measurements . . . . . . . . . . . . . . . . 19

2.4 Results . . . . . . . . . . . . . . . . 22

2.5 Tomographic Considerations . . . . . . . . . . . . 26

2.6 Sensor Design . . . . . . . . . . . . . . . . . . . 34

2.7 Electronic Requirements . . . . . . . . . . . . 35

2.8 Discussion and Conclusions ............. 36 
3. PROJECTIONS . . . . . . . . . . . . . . . . . . . . . . 39

3.1 Plans for Phase II Work . . . . . . . . . . . . . . 39

3.2 Beyond Phase II .................... . . 40

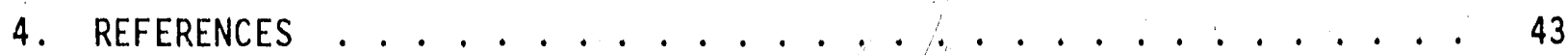

Appendix A - Calculations . . . . . . . . . . . . . . . A-1 


\section{FEASIBILITY STUDIES FOR THE SONIC SENSOR SYSTEM \\ FOR NON-INVASIVE TEMPERATURE MEASUREMENT \\ IN THE FOOD PROCESSING INDUSTRY}

\section{INTRODUCTION AND OVERVIEW}

\subsection{The Need for a Sensor}

\subsubsection{Conventional Methods}

Conventional canning methods have seen little change in the basic process in nearly 100 years. Conventional canning is a batch process having typical control steps as follows:

- Mix the food materials, fill, and seai the cans

- Retort in batches (typically several thousand cans) by heating to temperatures in the range of 120 to $150^{\circ} \mathrm{C}\left(250\right.$ to $\left.300^{\circ} \mathrm{F}\right)$ and holding for specified periods of time, typically 5 to $15 \mathrm{~min}$, primarily to kill harmful bacteria

- Puncture a representative number of cans and insert thermometer or thermocouple to determine internal temperature, as a process-control mechanism

- Cool to safe-handling temperatures

- Dispose of all sampled cans.

The key step is retorting. While this cooks the food, its primary purpose is the destruction of harmful organisms, most notably those responsible for botulism. The time-and-temperature regime for this is experimentally determined for each plant and process, but is subject to a number of practical constraints that introduce the need for safety factors. 
The iemperature measurement does not assess internal particulate temperatures, but serves only to verify that the process parameters are within acceptable limits. Particulates heat at a different rate from the liquid (mostly water) in wich they are processed. The heating rates are also affected by the degree of filling of the cans, particulate sizes and densities, and frequently by variations in parameters dependent on crop variety and season.

As a result, conservative safety factors are added to nearly every step in the process to ensure a safe product. Product quality is always degraded by overcooking, and a significant amount of excess energy is used; the excess is estimated at roughly $20 \%$ of that used in the whole canning process.

Further, punctured cans and their contents from the sampling process are wasted. A typical large processing plant will dispose of 100,000 sampled cans in a season. Increased disposal costs of wet waste are added to the cost of product, can, and processing.

\subsubsection{The Aseptic Process}

The aseptic process circumvents many of the limitations of conventional processing. Schematically, food materials are continuously pumped through a heat exchanger in which they are first heated to the required temperature, held for a prescribed period of time determined by the length of the heatexchanger tubing and flow rate, cooled (with heat recovery in a recuperator), then finally placed and sealed in pre-sterilized cans. Because the process is continuous rather than batch, it is more productive in cans/unit time/unit plant floor space. Modern aseptic processes typically use on $1 y 10$ to $20 \%$ of the energy of the equivalent conventional process, and produce significantly better product quality because they do not need to overcook the product. The aseptic process is used in the U.S. for prefucts consisting of a single-phase, mostly for fruit juices and similar high acid products sold in boxes or bags.

In single-phase materials, temperatures can be measured simply and accurately at any desired location in the heat exchanger by thermocouples or 
similar devices; the process is under good control. Note, however, that residence time in the heat exchanger pipe is not well defined, because the product that travels down the middle of the pipe can travel faster than the product closest to the wall.

This makes 1ittle difference when the temperature of all parts of the product can be easily measured, but it is a major difficulty when the product contains particulates. Because the internal temperature of particulates cannot be measured by conventional means and their residence time at processing temperature is not well defined, the Food and Drug Administration (FDA) has not approved the aseptic process for this use and assume that the process cannot be proven safe for particulate foods.

This dilemma can be resolved with a sensor that measures the internal temperature of particulates during processing.

A sensor capable of noninvasive measurement of the internal temperature of particulate foods would have application both to conventional and to aseptic processing. In conventional processing, such a device could replace the present destructive sampling method with one that is nondestructive, producing significant reduction in product waste and improved process control, potentially allowing up to $20 \%$ reduction in energy usage and improved product quality. Such a device would, in effect, enable the aseptic process to be used for particulate foods in the U.S., yielding energy savings up to $80 \%$ compared to present practice, with significantly improved product quality.

The feasibility of such a sensor is the subject of this report. 


\subsection{The Sontc Sensor System Concept}

\subsubsection{Measurement of Temperature by the Speed of Sound}

The approach to design of the sensor is based on speed of sound tomography. The speed of sound in any material, solid, liquid, or gas, is a strong and well-defined function of temperature. Typically, the speed of sound is measured by timing the passage of a short pulse of sound from a source or transmitter to a receiver through the medium whose speed is to be measured. The transit time is inversely proportional to the speed of sound and easily related to the temperature of the medium. Laboratory accuracies of a thousandth of a degree celsius under carefully controlled conditions are commonplace. This method is often used in industrial applications when the flight path is in a single medium.

The relationship between speed of sound and temperature is a specific property of each material through which the sound passes. Each material, for example, water and potato, has its own relationship between the two, and a given speed of sound may indicate one temperature in water and another in the potato. When the acoustic flight path of a single sound beam is partly in a liquid and partly in a solid, there is no general relationship between the total transit time and the speed of sound unless the fraction of the flight path occupied by each material is known. In food processing, the position and size of potato chunks along the sound beam are random, and it is possible to say only that the total ime of flight is proportional to the average speed of sound along the path, and little or nothing about the temperature.

This is a classical problem in application of ultrasound to food

processing. A recent review article by Javanaud discusses this problem. With the present ultrasonic practices in general, whether measuring the speed of sound or any other acoustic parameter related to the properties of the food material, the parameter of interest can be found only with great difficulty and then usually with inadequate accuracy, in a random two-phase mixture. Prior research approaches have used approximations applicable only to 
dispersions (presumes that the medium can be represented to an acceptable degree by a single phase). More general approaches have been directed toward developing for each measurement a specific model of the two-phase mixture that would have given the acoustic results actually observed in each measurement; this approach takes very large computing power, does not generally have a unique solution, and has not met with significant suscess even in the laboratory.

The problem is to ascertain that portion of the acoustic flight path that passed through each phase when the geometry is randomly different in each measurement. If the length of flighi path in each medium is known, the speed of sound, and thus the temperature, in each could also be known from the overall measurement of transit lime. It requires only a calibration in each medium.

\subsubsection{The Tomographic Process}

The tomographic process provides a way to determine the effective flight path in each medium. The process in fact provides a map or image of the cross section of the can or pipe being measured, from which the speed of sound in any small portion of it can be obtained. Tomography is a mathematical process by which measurements on a large number of individual flight paths are combined to produce a cross-sectional view or image of an object. The process works schematically as follows.

Imagine a large number of ultrasonic transmitters on the periphery of a pipe or can, all pointing in, and an equal number of receivers arranged so that the flight path between each transmitter and receiver combination follows a chord of the circle (Figure 1). Collectively, the ray paths intersect in a dense pattern throughout the cross section of the pipe. Suppose, now, we wish to determine the speed of sound in each of a series of squares, as shown in Figure 2. We arrange the geometry so that at least two rays from some source to a receiver cross each square. 


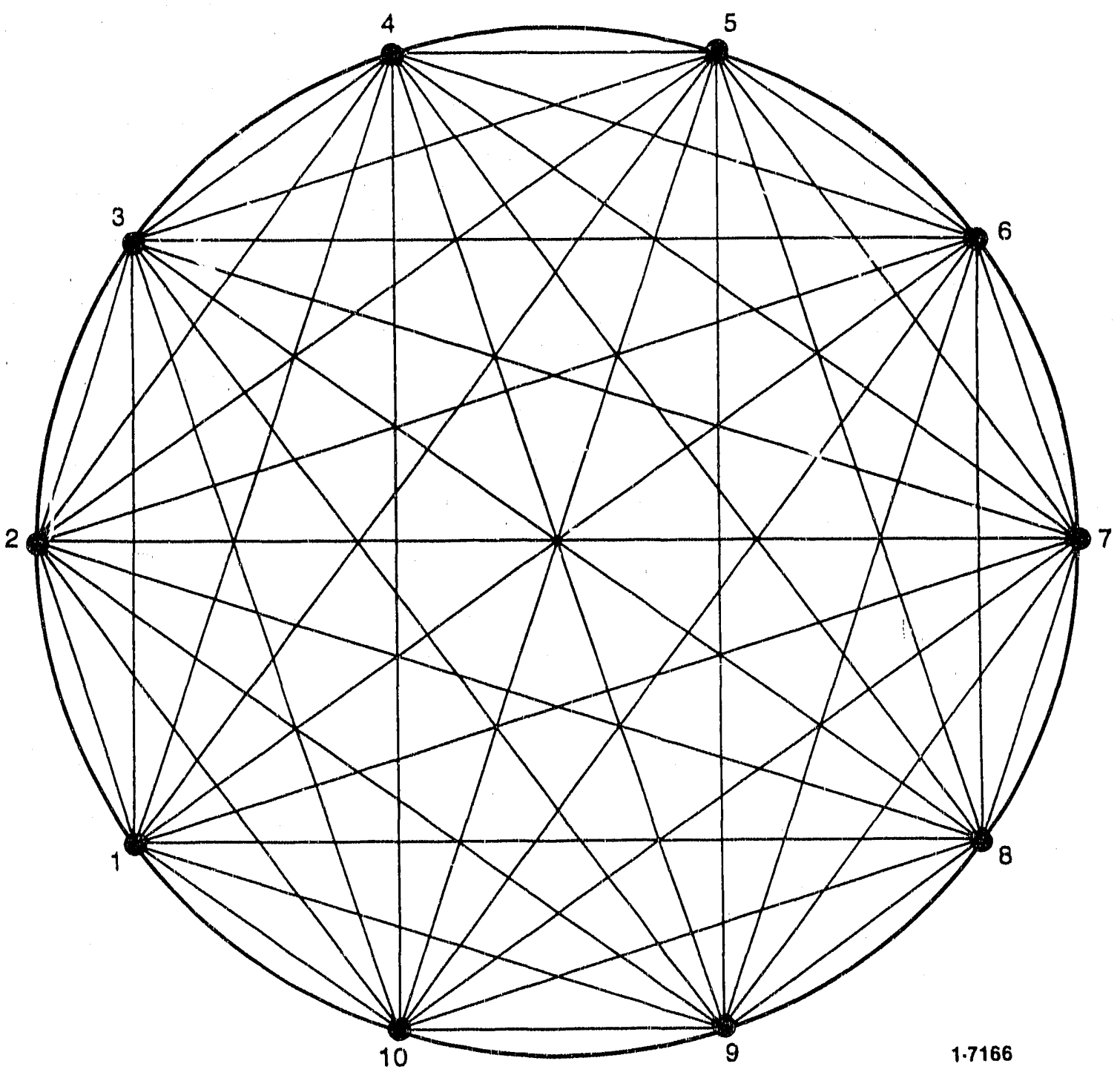

Figure 1. Idealized array for tomographic reconstruction. Each number:od dot corresponds to a transducer source and receiver combination. A measurement of transit time would be made along each of the rays shown between each transducer and all of the others. 


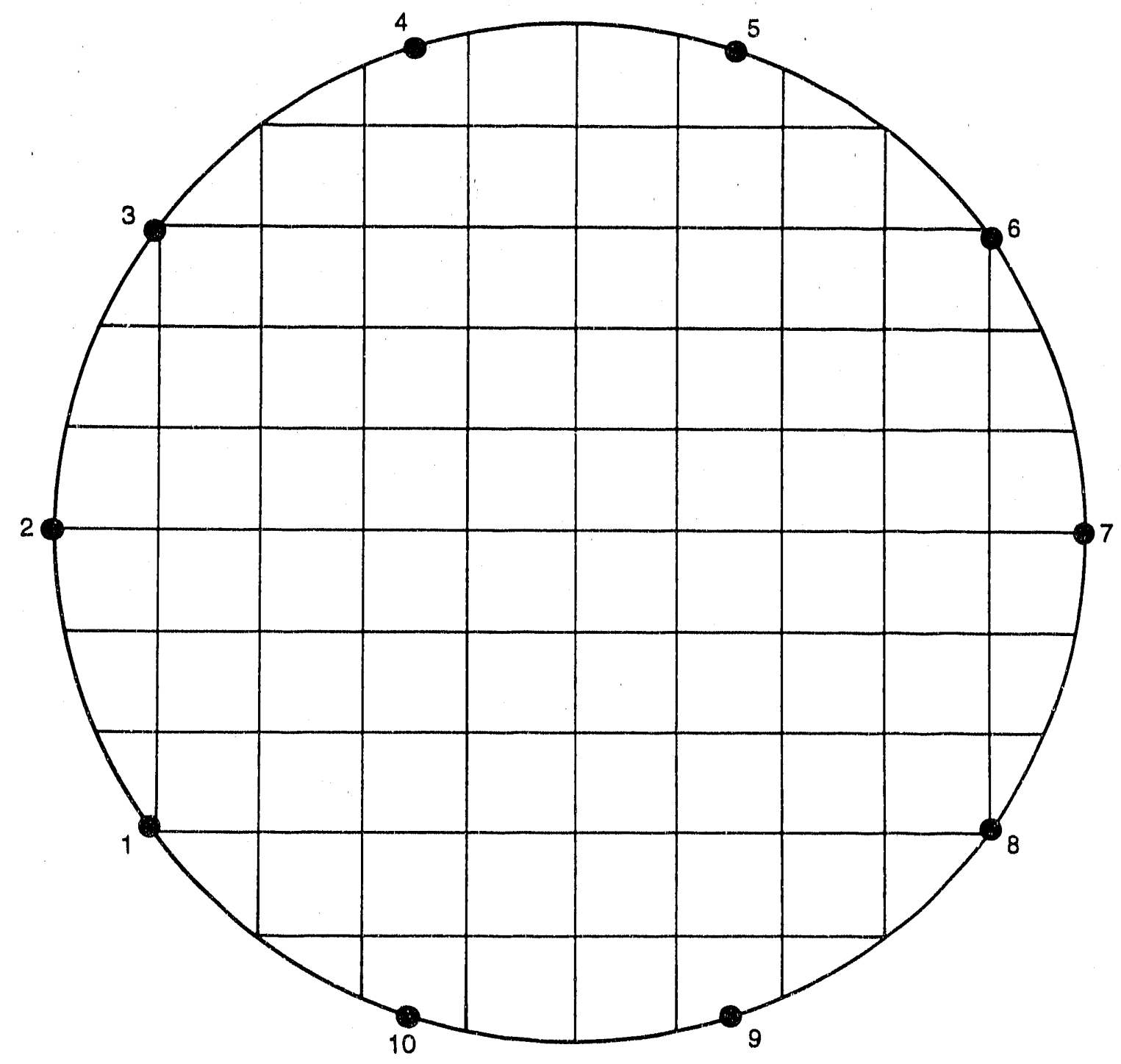

1.7165

Figure 2. A possible tomographic reconstruction grid. The objective is to determine the effective average speed of sound in each cell by tomographic reconstruction of the multiple measurements indicated in Figure 1. Each cell contains portions of two or more rays connecting sources and receivers in Figure 1. 
For each ray path, for example between transmitter 1 and receiver 6 , we can determine from simple geometry the iength of the ray path in each square, as shown in Figure 3 . The transit time across that square is the (known) path length divided by the (unknown) speed of sound in that square. By proceeding from square to square along each ray, an equation can be written for the overall transit time (which is measured) of the ray in terms of path length (known from the geometry) and transit time in each square (which is not yet known), and set it equal to the measured overall transit time.

Because two or more ray paths cross each square, and because the transit time in each square is a constant (though still unknown) for each ray path in each square, it is possible to write a series of linear simultaneous equations that can be solved by a number of methods for the transit time (or the speed of sound) in each square. To produce an image or map of the cross section of the pipe, simply assign a grey-scale tone or color proportional to the transit time value in each square. The result is a map or image of the transit time, and thus the temperature, throughout the cross section of the pipe, including the interior of any particulates traversed by the sound pulse.

The quality of the image depends on the sizes of the squares, that is, on how many squares there are in the cross section of the pipe. The image improves as the squares become smaller. The size of the square determines the spatial resolution with which the temperature is measured. Note, however, that smaller squares mean more ultrasonic sources and receivers, more accompanying electronics, and more overall complexity; other problems develop as we11. There is clearly a practical tradeoff among these parameters. One of the major objectives of the feasibility study has been an exploration of this tradeoff.

The image shows the location of any particle wherever it may be in the cross section. Particles can be identified simply by the fact that they are closed figures, while the interstitial liquid between the particles "snakes" around them. Identification can be accomplished by software without the need to produce an image. In the event that there is more than one class of 


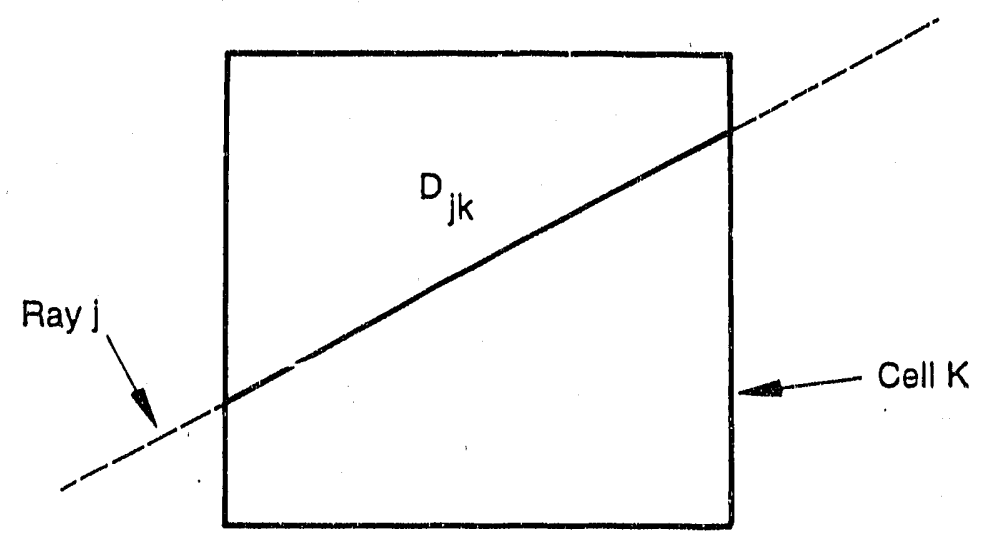

1.7157

Figure 3. Ray path through a representative cell. A portion of ray $j$ is shown passing through cell $k$ of Figure 2. The length of ray $j$ in cell $k$ is $D_{j k}$, which is a simple geometric property of the array. The total length of ray $j$ is the sum of $D_{j k}$ over all $k$ through which it passes betijeen source and receiver. 
particulates, as in beef stew, there are several ways to handle the identification or temperature measurement or both, al so by way of software.

Tomography has been well established in the medical field for many years, where it is the basis for medical computerized axial tomography (CAT) scans. In that case, the variable being measured is the attenuation of $x$-rays, instead of speed of sound or transit time, but the equations and the validity of the process remain the same. It makes no difference to the tomographic process what the physical variable is. Variations of the tomographic process have been used in a huge number of scientific disciplines, ranging from electron microscope studies of the molecular structure of bacteriophages, to studies of the earth's mantle using earthquake waves, to reconstructions of the $x$-ray structure of supernova remnants. In each case, the tomographic process is one of producing an image of a two-dimensional distribution of some physical variable from estimates or measurements of its integral (or average) value along lines of known location. ${ }^{2}$

Individual transit-time measurements can be made to uncertainties of $0.01 \%$ or less under industrial conditions. Uncertainties of this order correspond to errors in temperature measurements of tenths of a degree or less. When such measurements are combined in the tomographic process described below, uncertainties in measurement increase. There is an engineering tradeoff between the properties of the tomographic process, the complexity of the ultrasonic sensor and electronics, and the uncertainties of temperature measurement; one of the objectives of the feasibility study described in this report, therefore, was to explore this tradeoff for practical applications.

The concept of speed of sound tomography and its application to food processing is the subject of a patent application now being processed. Additional disclosures are in preparation regarding novel aspects of sensor design. This disclosure covers:

1. Use of speed-of-sound tomography for temperature determination in a particulate-containing liquid 
2. Use of combined through-transmission and scattering or reflection times of flight and signal amplitudes to determine physical properties of the liquid particulate constituents.

The patent application is based on the results of the feasibility studies covered by this report. Additional disclosures are in preparation regarding novel aspects of sensor design.

In summary, the basic technical concept combines speed of sound and tomographic technology in the following manner:

- Temperature is determined by speed of sound measurements. This is an average temperature along the sound path.

- Multiple simultaneous measurements from different directions are combined by tomography to assign appropriate sound speeds/temperatures to each portion of the container cross section. The data manipulation is similar to that involved in a medical CAT scan.

- Accessory equipment capability provides for automatic recognition and reporting of particulate temperatures. 


\section{FEASIBILITY STUDIES}

\subsection{Questions for the Feasibility Studies}

The Sonic Sensor System feasibility studies considered the following four questions, which were addressed experimentally.

1. Are the properties of food materials compatible with accurate measurements by this concept?

The major questions in this category relate to the speed of sound in particulate food materials as a function of temperature, the permanent changes in the speed of sound that may be introduced by cooking these materials, and attenuation or scattering of sound waves.

At the beginning of this study, the relevant acoustic properties of food materials were essentially unknown. For example, we could postulate gelling of the starch in such foods as potatoes, which might tend to invalidate the test as a reliable measure of temperature because it can cause the speed of sound to change without an actual change in temperature.

These questions were addressed experimentaily.

2. Are the requirements of the tomographic process in ultrasound compatible with the size and geometry of a can or pipe and with a feasible ultrasonic sensor system?

The geometry required for a tomographic uitrasound measurement on a can or pipe is unusual in that the source and receiver for each ray essentially must touch the volume sampled. This limits the number of ultrasonic transducers which have lower size limits set by wavelength considerations. The tomographic process itself introduces uncertainty (noise) into the process. This kind of noise depends on the number of rays, their geometry, 
and on the tomographic algorithm used. Real electronic noise is a serious additional complication.

Initially, it was not clear if this question had practical affirmative answers for the requirements of a Sonic Sensor System. This problem was addressed mathematically using a number of tumographic reconstruction algorithms and Monte Carlo calculations to investigate notse problems, resolution, accuracy, and ray-number requirements.

3. Is it feasible to design and build a sensor system that would be compatible with the size requirements?

Initial calculations of tomographic properties, as in the last paragraph of 2.1.2, indicated that Sonic Sensor system requirements could probably be met with the number of sensing rays in the range between 50 and possibly several hundred different ray paths through the pipe or can cross section. This is a large number, considering that there is a finite lower-size limit for ultrasonic transducers, which is set by the wavelength of sound (typically of the order of 1 to $3 \mathrm{~mm}$ for this type of measurement.) The feasibility of cramming the required number of components into the allowable area or volume was initially in question.

This problem was approached with an engineering conceptual design study based on previous INEL experience with unusual ultrasonic requirements.

\section{Are the electronic systems feasible to meet the measurement requirements?}

The two sub-questions are: (a) would such a system require development or use of unusual circuitry? and (b) would the complexity or number of circuits required price the system Deyond practicality?

The first question was approached by straightforward analysis of measurement and time requirements. We determined that commercially-available electronic circuitry can handle all requirements; however, the cost depends strongly on the speed at which repeated measurements must be made. For 
example, a standard personal computer would be adequate if measurements were needed every few seconds to a minute or so, but considerably more expensive computer hardware would be required if measurements were to be required on significantly faster time scales. Costs for the additinnal support and signal electronics were found to vary similarly.

The answer to the second question depends on both the number of ray paths required by the tomography and on the sensor configuration itself. This will be discussed in more detail in Section 2.5 - Tomographic Considerations and Section 2.6 - Sensor Design.

Technical feasibility of the concept rests primarily on qualitative aspects of the questions addressed above. 


\subsection{Experimental Measurement of Acoustic Properties of Food MATERIALS}

\subsubsection{Test Material}

Foods are mostly water. For example, potatoes are typically more than $90 \%$ water by weight, and most other vegetables are roughly the same. Meats have less water, but water is still the major constituent. In each case, the solids contain and supply a framework to hold the water.

One could therefore expect that sound will be propagated primarily through the water component of the food material structure, but that prop jation would be modified by scattering and other interactions with the solid part of the structure. In the absence of changes in structural properties caused by cooking, it could be expected the speed of sound would be a few percent higher than that of water at any temperature. The speed of sound in water at room temperature is approximately $1.5 \mathrm{~km} / \mathrm{s}$.

The speed of sound in water has its maximum value at a temperature slightly over $70^{\circ} \mathrm{C}$. Sound in 1 iquid water is slower at both higher and lower temperatures.

The effects of the solid part of food materials, and the magnitude and sign of changes induced by temperature and by cooking, were the major focus of the experiments.

Potatoes are considered a worst case from these viewpoints because the starch content of potatoes is a major constituent that is known to change its mechanical properties, and thus the speed of sound, when heated in water. There are a number of different starches in foods, but nearly all have gelled completely by the time they have reached $90^{\circ} \mathrm{C}$. We considered that if the acoustic properties of potatoes lend themselves to measurement by the Sonic Sensor system concept, so would the properties of nearly all food materials. 


\subsubsection{Apparatus}

Typlcal food-processing temperatures are upwards of $120^{\circ} \mathrm{C}$. Pressurized apparatus was required to achleve these temperatures in experiments.

The experimental apparatus was adapted from a conventional home pressure cooker, heated by a commercial $4 \mathrm{~kW}$ electric hot plate. The hot plate dial settings provided an excellent range of heating rates for all testing. The cooker was modified to pass coaxial cables for ultrasonic transducers and a thermocouple through the pressure boundary. An internal fixture positioned the food material and transducers for through-transmission of ultrasound over an adjustable-length experimental path (25 to nearly $125 \mathrm{~mm}$ ).

The thermocouple was embedded in the center of the food material to read its temperature. An external pressure gage permitted a thermodynamic determination of the temperature of the water within the cooker, based on pressure. Appropriate signal-generating, receiving, conditioning, and datarecording equipment completed the test set-up. A block diagram (Figure 4) identifies a11 components.

Test sensors were special high-temperature uitrasonic transducers operating at a nominal $750 \mathrm{kHz}$ center frequency. This frequency was chosen from experience with difficult-to-penetrate materials often found in nondestructive testing applications. The effective diameter of the sound beam for these measurements was $15 \mathrm{~mm}$, significantly greater than would be used in a food-processing system.

Apparatus modification to accept a metal-sheathed, ceramic-insulated cable, with accompanying changes to the interial fixtures, was found necessary; standard coaxial cables simply melted at the temperatures of the experiment. 


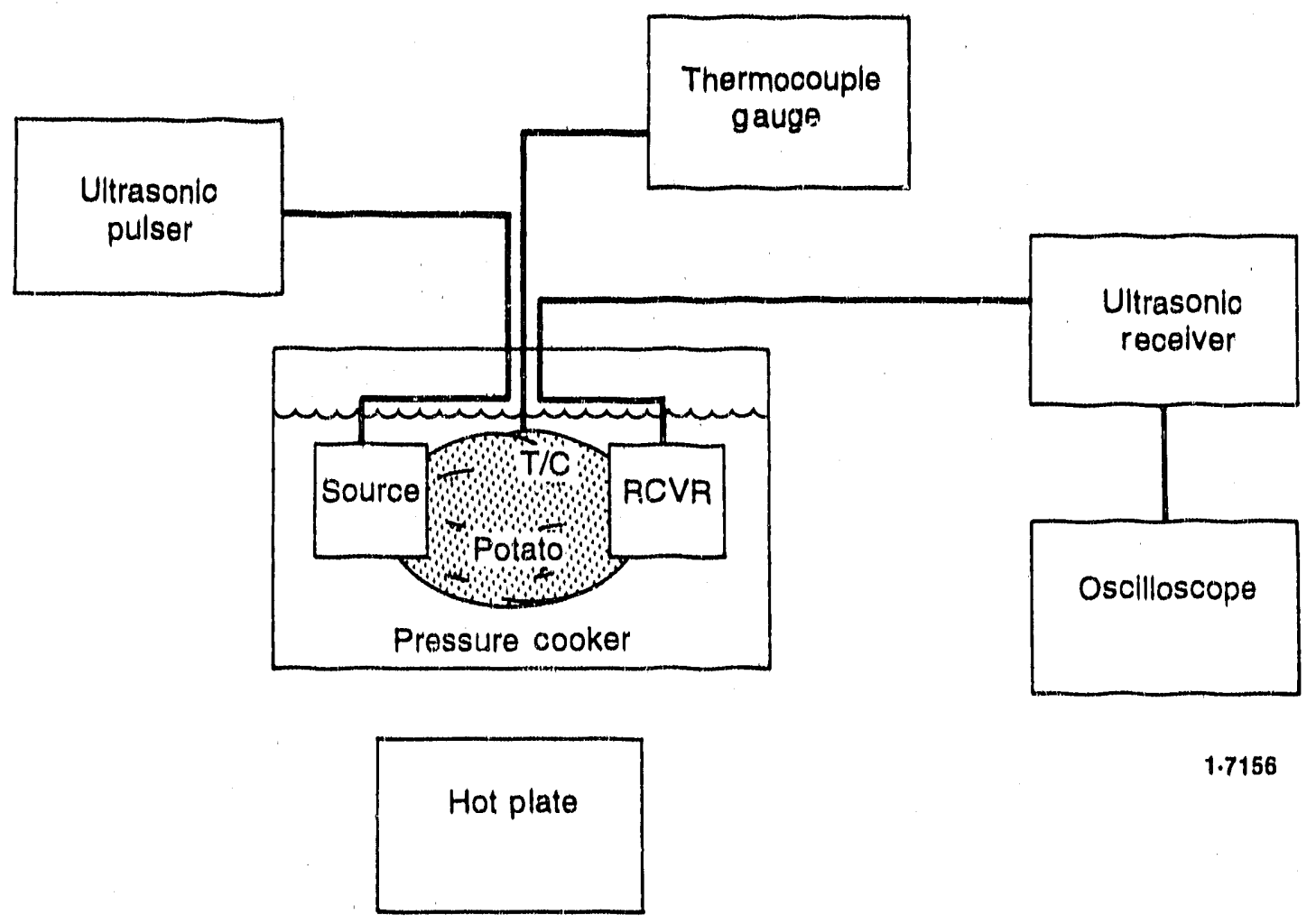

Figure 4. Schematic block diagram of experimental apparatus. 


\subsection{Measurements}

For calibration, the cooker was completely filled with water alone. The heating rate for this condition was almost independent of the water temperature (measured by the thermocouple), but depended on the hot plate control dial setting as shown in Figure 5. The 22-psig relief valve on the pressure cooker permitted the apparatus to reach $122^{\circ} \mathrm{C}$.

Measurements wore made relative to the sound pulse transit time through a referance material (water) at a reference temperature (nominally $20^{\circ} \mathrm{C}$ ), to provide calibration for inter-transducer spacing. Figure 6 shows a typical calibration for a $65-\mathrm{mm}$ spacing. The transit time shows a minimum (maximum sound speed) at $73^{\circ} \mathrm{C}$; measurement precision for this figure is $-0.22^{\circ} \mathrm{C}$.

UTtrasonic sensitivity of the system over the temperature range used showed only a slight change, dropping $\angle 5 \%$ at $115^{\circ} \mathrm{C}$. In water, which has low ultrasonic attenuation, both the through-transmission (one round trip) signal and succeeding pulse trains (making one or more round trips between transducers acting as mirrors) are detected. Up to 12 such pulse trains were observed in this work, indicating good signal/noise ratio (SNR) and alignment.

Subsequent measurements were made with potatoes in place, over a range of temperatures $\left(20\right.$ to $\left.120^{\circ} \mathrm{C}\right)$. Transducer placement was such that the external water acted solely as a heat-transfer medium; the effective sound path was entirely in the potato.

Three varieties of potatoes, White Rose, Red Rose, and Idaho Russet, were examined. The Idaho Russets were from two different crop years. 


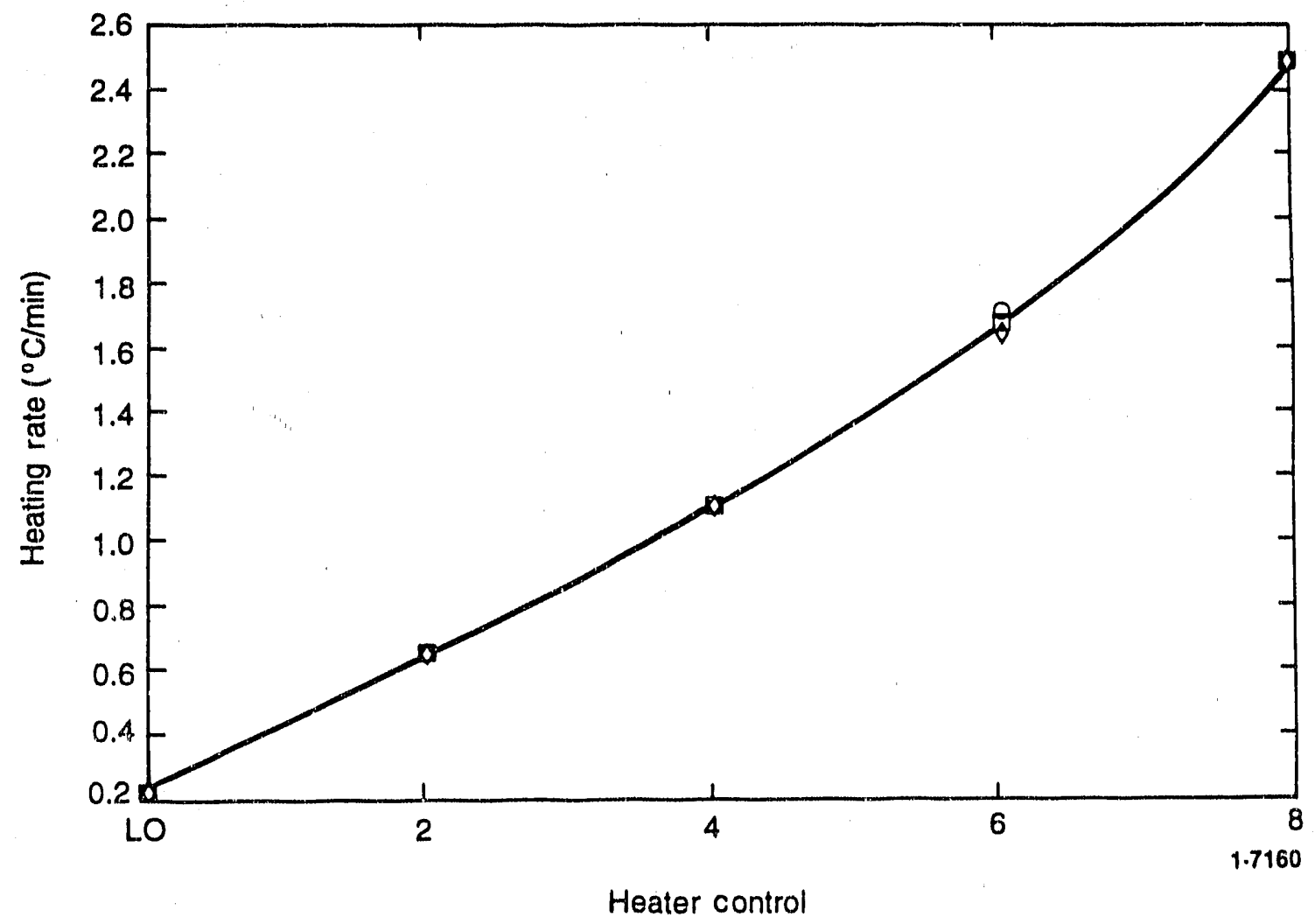

Figure 5. Heating rate of the experimental system filled with water only. 


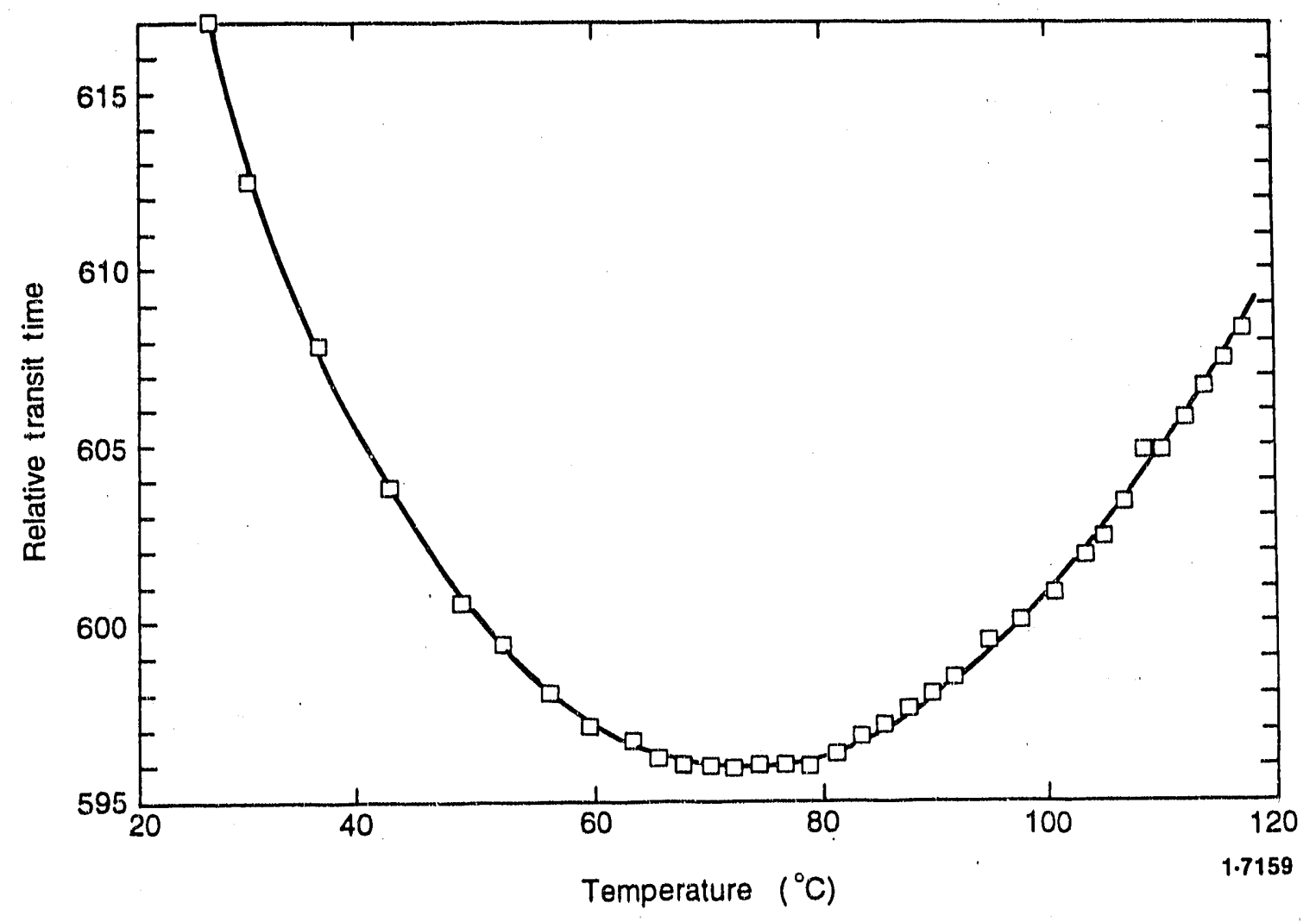

Figure 6. System calibration in water. The source/receiver spacing is $65 \mathrm{~mm}$. The transit time (abscissa) is in arbitrary instrument units. 


\subsection{Results}

Figure 7 presents typical transit time versus temperature results for tested potatoes. The curve of transit time versus temperature has three regions: (a) temperatures below $-50^{\circ} \mathrm{C}$, where the exact shape of the curve depended on heating rate, (b) the temperature region between $-50^{\circ} \mathrm{C}$ and 100 to $110^{\circ} \mathrm{C}$, where the shape apparently depended on starch gelling characteristics and the heating rate (to a lesser extent), and (c) temperatures above $-110^{\circ} \mathrm{C}$, which was essentially independent of heating rate.

The shape of the curve starting at room temperature depended on the heating rate, which closely approached the curve for water in this regime at low heating rates uritil the internal temperature reached $-50^{\circ} \mathrm{C}$, while departure from the shape of the water curve was marked (as shown in Figure 7) when heating rates were high. The reason is that thermal gradients at high heating rates left low temperatures in the center of the specimen where the thermocouple was located. The sound beam sampled a region that did not have a uniform temperature. At low heating rates, the thermal gradients were smaller, and the temperature of the potato more closely approached that of the heating water.

The shape of the curve was definitely nonreproducible in the intermediate temperature range, and it generally did not have the smooth parabolic shape shown in Figure 6 for water. This is interpreted as showing that the intermediate temperatures are the region where starch gels, and gelling depends explicitly on the starch content and the details of the timetemperature history of the potato, which in turn depends on thermal gradients.

The shape of the curve above $\sim 110^{\circ}$ was quite reproducible, and within the accuracy of this experiment did not depend on heating rate or time-temperature history. By the time the specimens had reached this temperature range, the starch had completely gelled and the potatoes were "cooked" and much more permeable to water than at lower temperatures, leaving essentially no thermal gradient within the potato. 


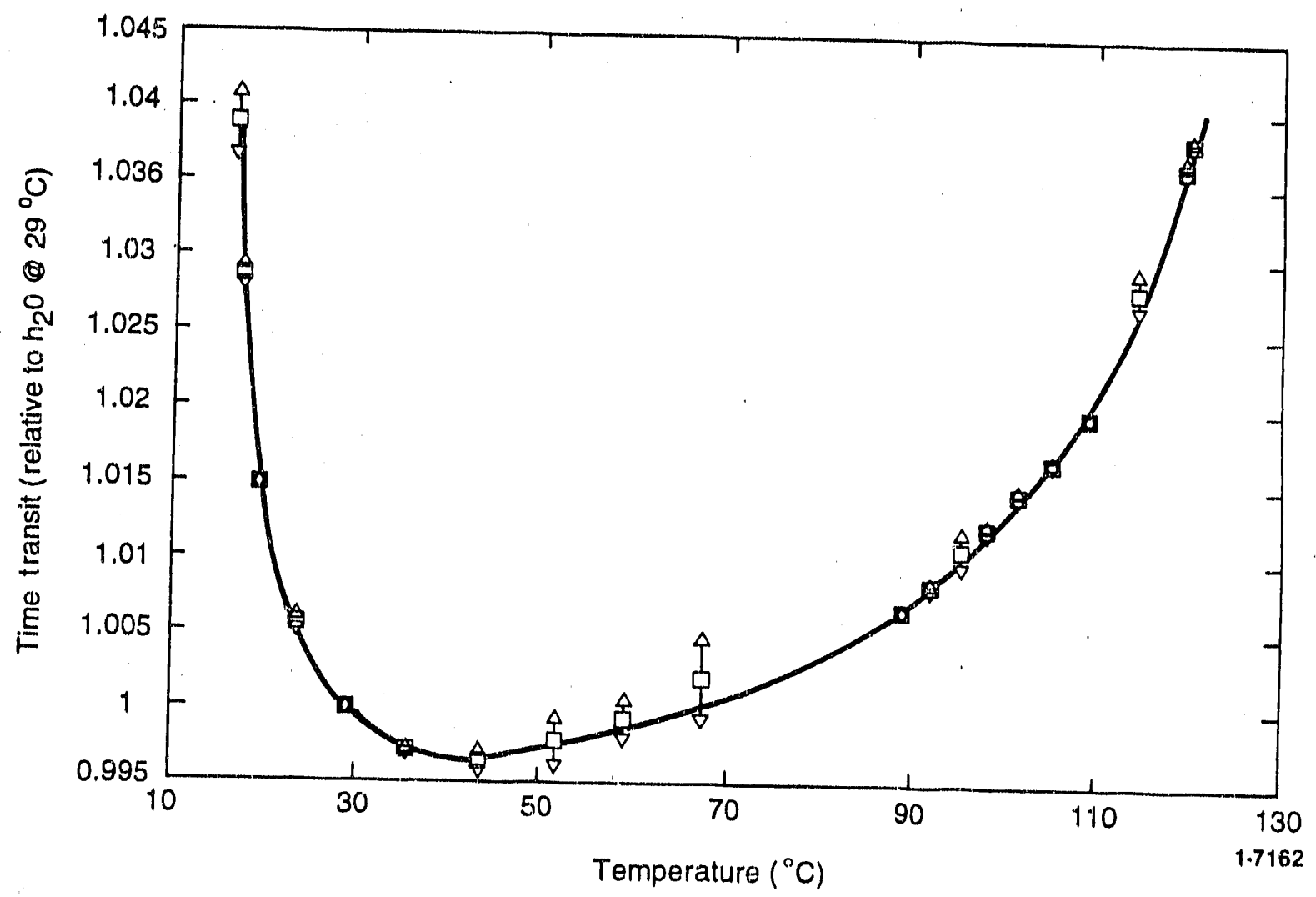

Figure 7. Typical transit time measurement in potato. The ray path was $-65 \mathrm{~mm}$, entirely in a potato. The transit time (abscissa) is plotted relative to that in water at $29^{\circ} \mathrm{C}, 1.51 \mathrm{kM} / \mathrm{s}$. 
It is important to note that the transit time versus temperature curve reaches the region of stability at temperatures below those used for food processing, which are generally at $120^{\circ} \mathrm{C}$ or significantly higher.

It is also important to note that thermal gradient effects indicated a lower temperature for the potato than it actually had. This is in the safe direction. Further, specimen size would be smaller in real applications, and sound-beam diameters would also be smaller; both of these considerations reduce the measurement effects caused by thermal gradients.

A permarient increase in transit time (amounting to a few percent), was found in samples on cooling to room temperature after heating. The effect is interpreted to be attributable to a hardening or strengthening of the structure by gelling of the starch. The hardening of the structure increases the speed of sound. The effect had been expected, but it was encouraging to find that it was this small.

No consistent or significant differences among potato varieties were noted within the accuracy of this experiment. However, a major effect relating to water content was noted. It was found that when the water content of the uncooked potato during storage dropped below some threshold value (which was not determined), sound would no longer pass through more than a few millimeters of potato. It was also found, however, that an acoustically opaque potato became transparent to sound again when its temperature in water was raised to $<50^{\circ} \mathrm{C}$. The transition was abrupt, and occurred in less than ten seconds. Apparentiy, heating forced sufficient moisture into the potato to provide for good propagation of sound.

With regard to the acoustic properties of potatoes versus temperature, two conclusions can be drawn from the above:

1. Sound propagation in this (high-moisture-content) medium is similar to that in a two-phase mixture, such as sand and water at the bottom of a fish bowl. To clarify:

- Sound is propagated primarily by the water. 
- The speed of sound of the potato is only a few percent higher than that of water alone, and is primarily determined by the properties of water.

- The density factor in acoustic impedance is determined by that of the solid, in this case the potato. The presence of this solid in the sound propagation path modifies the speed of sound of the potato water content, but only slightly, as noted above.

- Sound scattering by the solid makes the material effectively dispersive of sound speed as a function of frequency. This does not affect temperature measurement directly, but needs to be taken into consideration in sensor design.

- Potatoes do not attenuate the sound beam sufficientiy at processing temperatures to be a significant problem.

2. Starch gelling hardens the food material structure (thereby raising the speed of sound), but this change is completed at temperatures well below those of interest in food processing. The curve of speed of sound versus temperature for potatoes at processing temperatures approximately parallels that of water above $-110^{\circ} \mathrm{C}$, and has adequate slope and reproducibility to permit temperature measurements of good resolution and accuracy.

The first conclusion confirms the starting hypothes is concerning the way in which sound is propagated in potatoes. By inference, essentially all other food materials can be expected to have similar properties with respect to the speed of sound tomography because they have structures that are similar with respect to the propagation of sound.

The second conclusion is more important. It says that the properties of the worst-case food material are such that the concept is feasible. Essentially all fruits and vegetables can be expected to have properties that 
are closer to those of water, because most do not have the added complication of starch.

The ideal case would be one in which the speed of sound in the food material is the same as that in water; a single measurement sound beam would suffice, because the mixture would be effectively a single-phase medium with respect to the parameter being measured. The closer the situation approaches this ideal, the easter would be the system design problem. Conversely, the most difficult design problems would be those in which the speed of sound in the food material greatiy differs from that in water, requiring more accuracy and better resolution.

It can be concluded from these experiments on the worst case, potatoes, that the acoustic properties of food materials as a function of temperature in water do not present any barrier to the Sonic Sensor System; acoustic properties of food materials will permit good measurements by the speed of sound method.

\subsection{Tomographic Considerations}

It was necessary to determine how spatial resolution and the measurement of temperature depend on the number of transducers, on the number of ray paths through the pipe or can, and on certain forms of noise inherent in the measurement and tomographic methods. Each of these parameters affects the practicality and feasibility of design.

A large number of algorithms for performing tomographic reconstruction, that is, obtaining an image by the tomographic process, are known and in use. Each has its own advantages for one or more applications, but there is no known general analytical method for determining the "best" algorithm for an arbitrary application. To perform experiments that would adequately cover the required range of parameter and algorithm variations that needed to be investigated was not practical. The approach, therefore, was to perform numerical "experiments" by building and varying a mathematical model of a 
typical sensor system, then using results of the model to derive guidelines for later engineering tradeoffs in actual sensor design.

Any tomographic reconstruction algorithm has a certain amount of what is called modelling noise associated with tt. The modelling noise is related to the fact that the reconstruction squares or other descriptions on which the image is based (Figure 2) are finite, and the shape of an arbitrary food particle cannot be exactly represented by any combination of squares. The smaller and more numerous the squares, the less modelling noise results, but, at the same time, the more susceptible the image becomes to the effects of real electronic and measurement noise. Spatial resolution, the smallest volume over which the speed of sound is averaged to obtain its temperature, follows the same general rules as does modelling noise. Spatial resolution affects sensitivity of the measurement of the speed of sound and hence the accuracy of the temperature measurement. Noise of any kind affects both sensitivity and accuracy.

Because sensitivity, accuracy, and low susceptibility to noise cannot be maximized simultaneously, the optimum design for any given purpose involves compromise. The purpose of this work was to develop the information on which an optimum compromise can be made.

The numerical experiments first addressed the way in which modelling noise (which is an inherent property of each tomographic algorithm) changes with the parameters of the mathematical model for the numbers of ultrasonic transducers that appeared to be physically reasonable for the size and geometry of a temperature sensor. Then spatial resolution was calculated for models, algorithms, and ray combinations that tended to minimize modelling noise. Finally, Monte Carlo calculations were used to estimate the effect of measurement noise. This is the equivalent of asking what the signal/noise ratio of the final electronic system must be. The result is a series of curves to guide any final design.

A resolution curve is shown in Figure 8 for an array similar to Figure 1. This Figure shows how the spatial resolution (which affects sensitivity and 
accuracy) depends on the number of ultrasonic transducers for the geometry of Figure 1, where each transducer is connected to all of the others by measurement rays, and transducers are equally spaced around the pipe. Resolution of $4 \mathrm{~mm}$ can be achieved in the model with 40 transducers. This is an important number, because it borders on the maximum number that can be convenientiy arranged on a $75-\mathrm{mm}$ diameter pipe or can. This number also affects the overall system costs, because each transducer must have electronics that are relatively expensive.

Typical noise studies are shown in Figures 9, 10, and 11. In this sequence, the Monte Carlo calculation randomly placed five circular "potatoes" with speed of sound $1 \%$ over that of the water (background) in the pipe. (A $1 \%$ error in measurement of the speed of sound corresponds approximately to a six-degree error in measurement of temperature.) The figures are histograms representing the number of grid squares in which the model calculated a speed of sound at a given fraction of that of the water. Each figure represents the results of 500 different random placements of the five potatoes in the measurement grid.

There was no (simulated) electronic noise in Figure 9. The width of the spikes representing water (at a value of 1.0 for the speed of sound) and potato at 1.01 are measures of the modelling noise. Figure 10 shows the effects of an addition of $0.05 \%$ random electrical noise, and Figure 11 represents the random addition of $0.10 \%$ electrical noise. The usefuiness of these calculations lies in the fact that they define the electronic SNR that must be achieved to obtain a given temperature resolution with the reconstruction algorithm and grid that was modeled. In this particular case, the reconstruction algorithm and modeled grid will require an SNR of 40 to $45 \mathrm{~dB}$ for good temperature resolution. This establishes SNR as a critical parameter, because SNR of this order approaches the practical limit. 


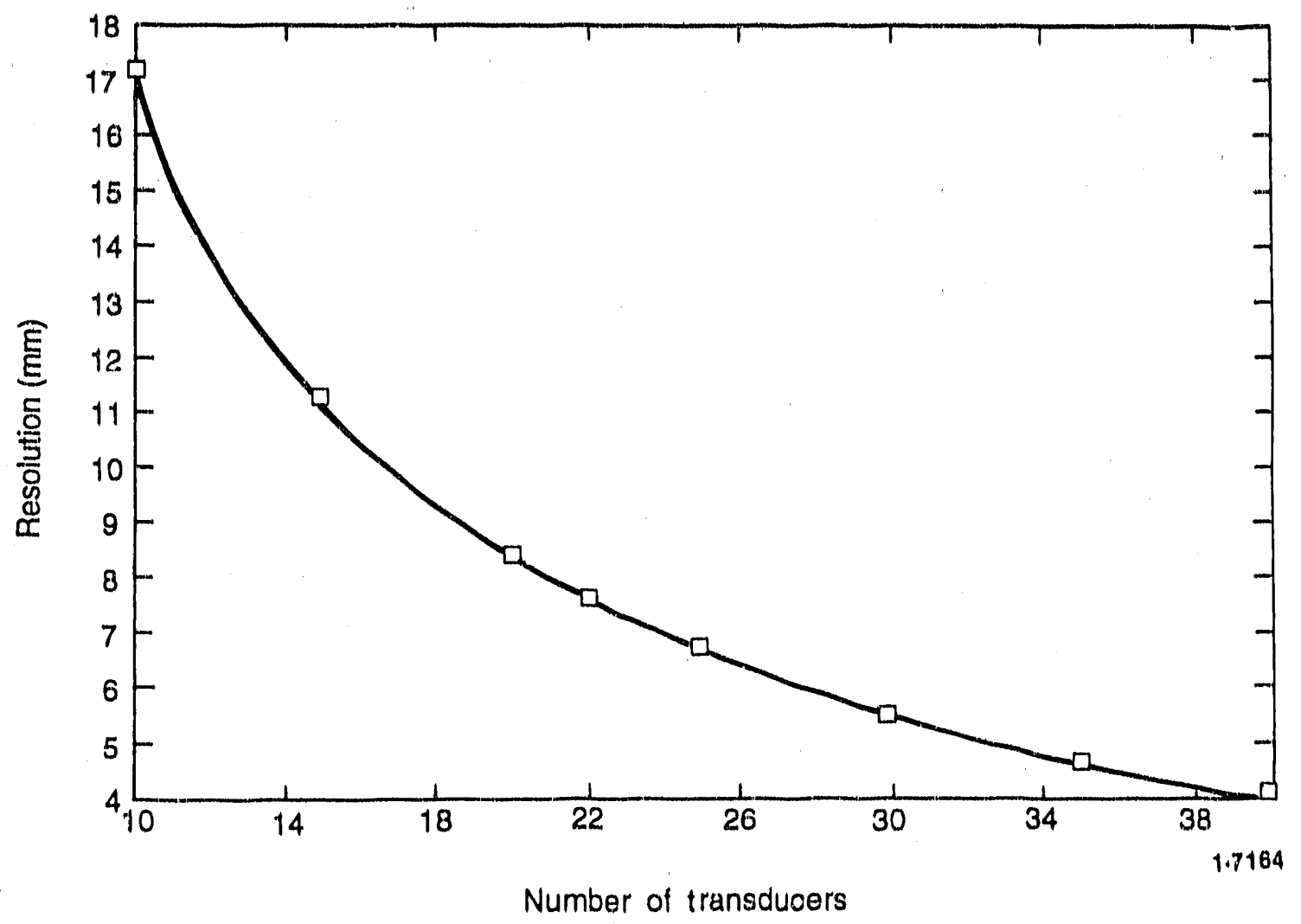

Figure 8. Calculated spatial resolution as a function of number of transducers. 


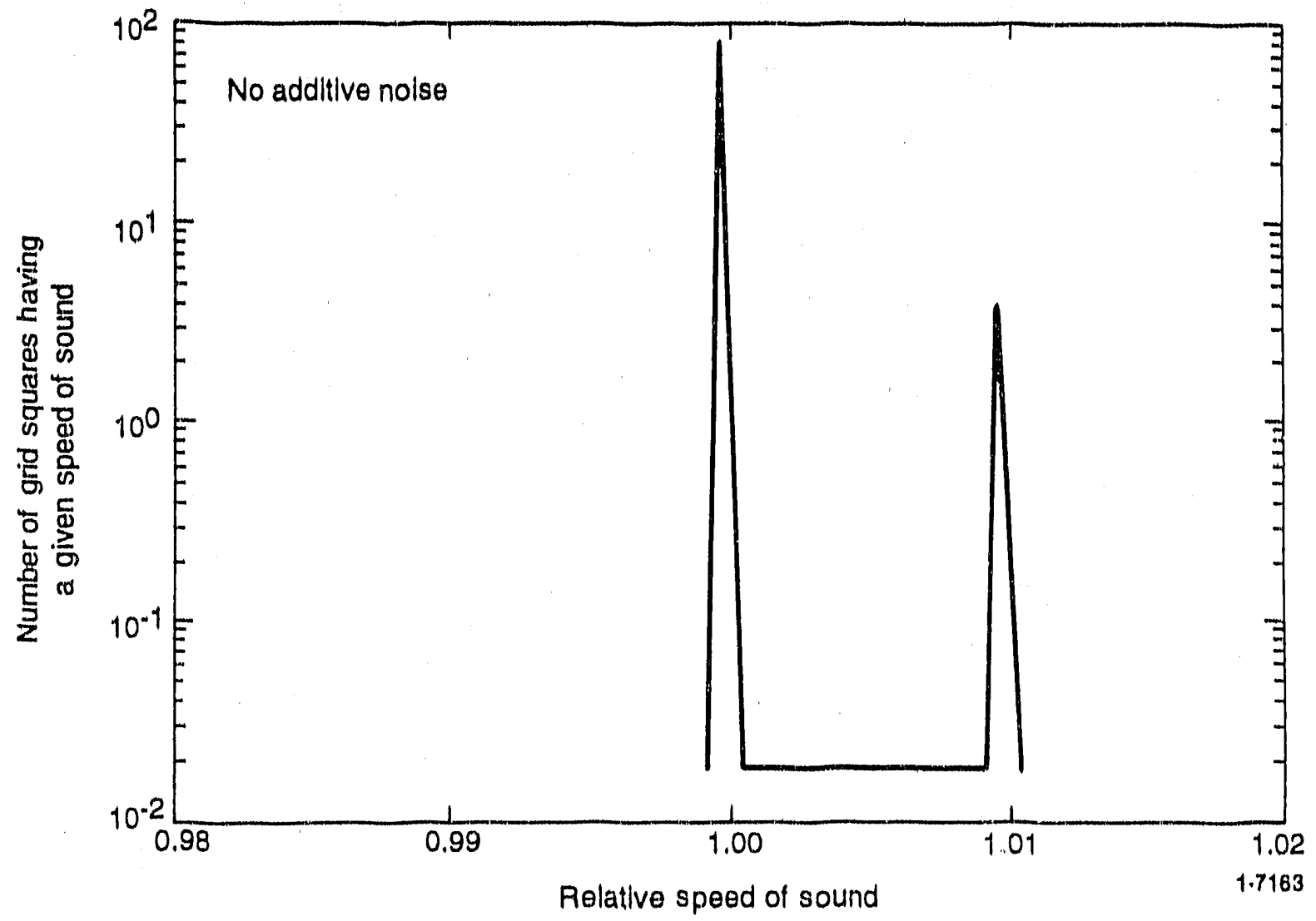

Figure 9. Estimate of the effect of reconstruction noise. The plotted data represent Monte Carlo calculations of the results of 500 random placements on the reconstruction grid of five circular "potatoes" having a speed of sound $1 \%$ greater than that of the surrounding water. The graph shows the number of cells having a given speed of sound (abscissa) plotted against the relative speed of sound. The speed of sound in water is taken as 1.0 . 


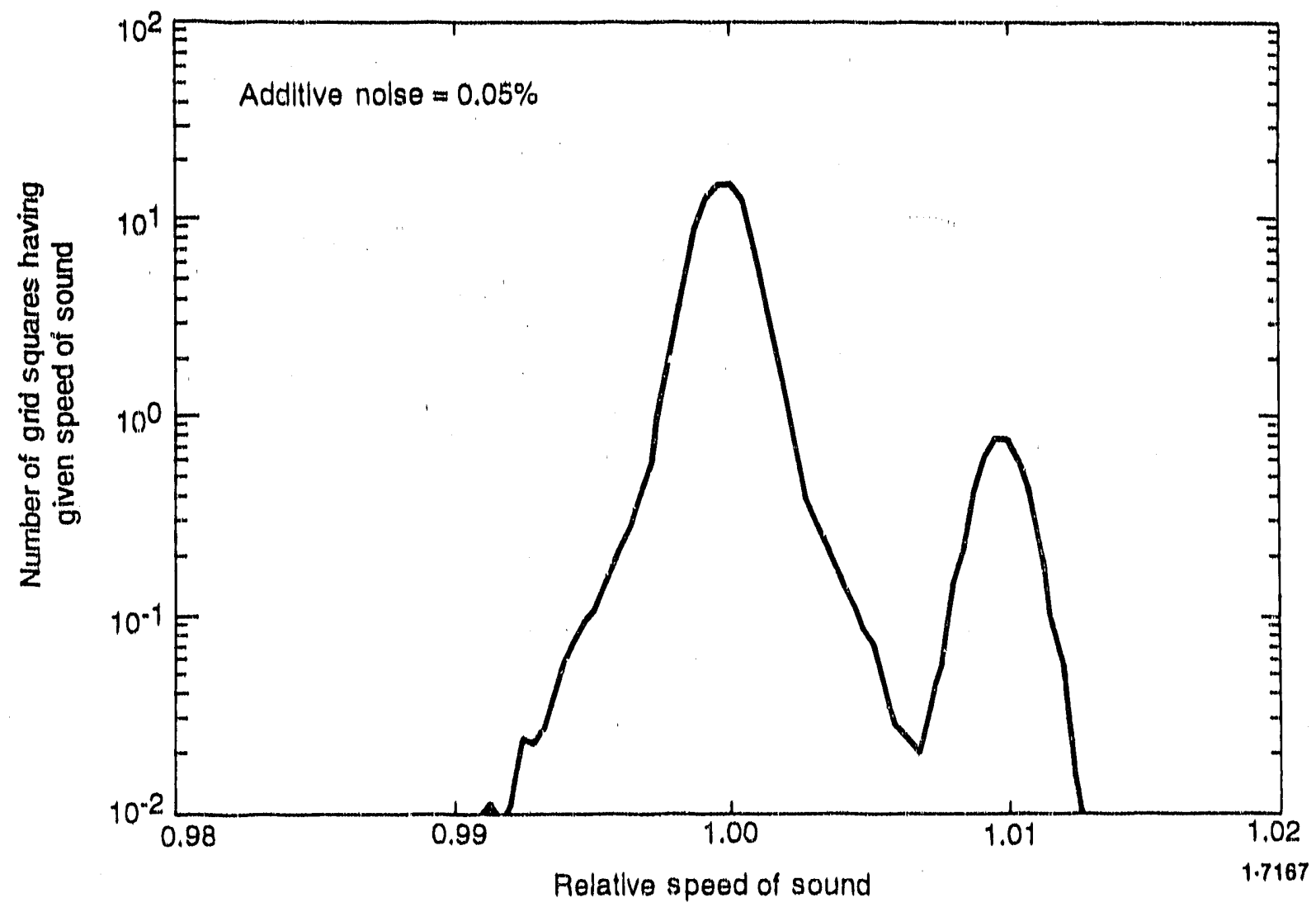

Figure 10. Effect of small amount of additive random electronic/measurement noise. The situation is the same as in Figure 9, except that $0.05 \%$ random noise has been added. 


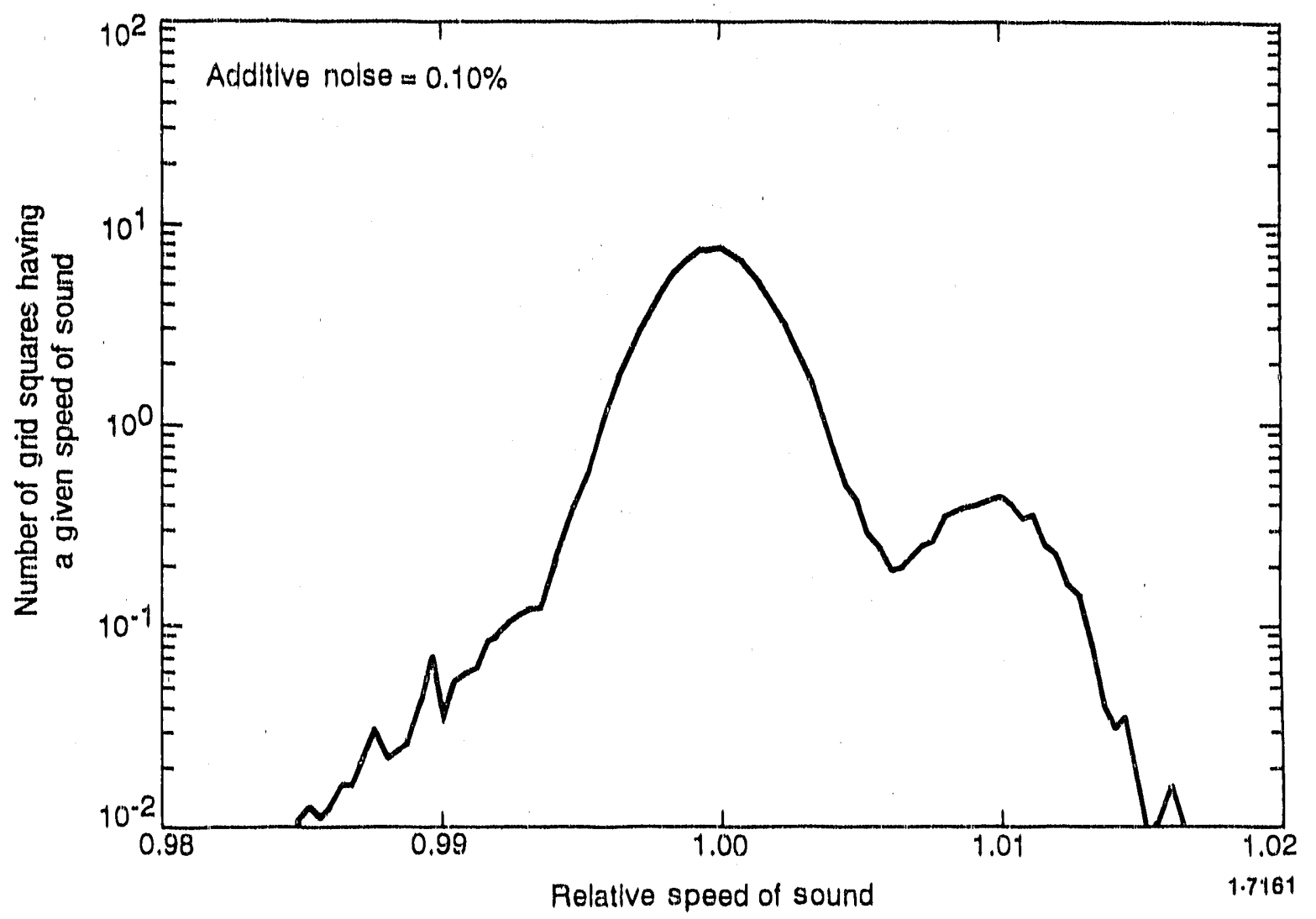

Figure 11. Effect of larger additive electronic/measurement noise. The situation is the same as in the previous two figures, except that random noise is now $0.10 \%$. 
It appears possible to perform a number of engineering tradeoffs during design that can relieve the situation. Other combinations of grid and algorithms may be less sensitive to electrontc noise, in effect trading an increase in modeling notse (which is small in this example) for less sensitivity to electronic noise in order to obtain a better optimum. Tradeoffs involving the ways in which the electronic systems measure transit time can also reduce the sensitivity to noise.

The conclusions of the tomographic studies are:

1. A number of available algorithms are appropriate for this design problem.

2. Adequate spatial resolution should be achievable with practical numbers and sizes of uitrasonic transducers

3. Modelling noise can be minimized, within the limitations of practical algorithms and ultrasonic transducer sizes and numbers, to obtain adequate measurement sensitivity and accuracy

4. SNR is a critical design parameter, the magnitude and effects of that must be handled carefully in the design process.

Combined, these conclusions show the feasibility of the concept from a tomographic reconstruction viewpoint. A number of solid engineering problems stand between this point and a final design, but each of these design problems appears to be within the present state of the art. It is important to note that the requirements of a practical sensor system for spatial and temperature resolution have not yet been established. It may well turn out to be the case that resolution requirements can be relaxed for specific food materials; in any event, establishment of the real requirements for these parameters is a prerequisite to beginning of the design process for a practical unit. 


\subsection{Sensor Design}

The most significant sensor design problem is achieving a sensor array analogous to that of Figure 1 , using practical transducers within the confines of a $75-\mathrm{mm}$ pipe or can. An auxiliary design constraint is minimizing the number of electronic elements needed to produce and detect sound pulses, a practical problem affecting overall costs and reliability of a system. A separate design study addressed these issues.

Any practical system will use piezoelectric transducers to generate short pulse. of ultrasound for the measurement and to detect these pulses after transiting the measurement volume. The transducers themselves are ceramic elements that must be provided with an electronic pulser to cause them to generate the sound pulse, and a receiver to detect the pulses. Additional electronic elements perform the measurement of elapsed time. Optimum transducer size is a function of its frequency, and size affects sensitivity (the larger the transducer, the more sensitive it is) and resolution of the receiving transducer (the smaller the better).

Two major issues were addressed. First, is it possible to appropriately arrange the required number of transducers having the required properties around a $75-\mathrm{mm}$ circle? The answer is that an appropriate array is relatively easy to achieve.

The second issue is more complex. The design problem can be illustrated as follows. Suppose the array is implemented with one pulser and one receiver/transducer per ray path; an analog-to-digital-converter (ADC) or other equivalent device and computer input circuitry would be required for each. This soon becomes unmanageable for a large array, because each transducer, pulser, and receiver can be expected to cost several hundred dollars, and ADCs may cost $\$ 1000$ or more each. Installation, wiring, software complexity, and reliability would be nettlesome and create expensive problems. The design problem is then one of minimizing cost and complexity, maximizing reliability, and optimizing performance. 
A design approach was found that minimizes the number of electronic elements and approaches the idealized geometry of Figure 1. The approach is based on the use of acoustic lenses of stainless steel cut into the pipe measurement section to define the geometry of source and receiver beams (circumventing a part of the transducer size/sensitivity tradeoff), and acoustic delay lines to minimize the number of electronic receiving and analog-to-digital-conversion elements. The delay lines direct signals from a number of locations in the array into a single transducer, each signal delayed by a fixed increment of time. A single receiver and ADC would thus provide for measurement of a number of rays. Production of the required geometry would use standard machining or casting techniques or both. The details of this concept are sufficiently unique that they are the subject of a second patent disclosure.

The sensor thus appears to be a straightforward, but intricate, design problem. From a sensor viewpoint, the concept is feasible.

\subsection{Electronic Requirements}

All portions of the signal generation, acquisition, and conditioning problem required to implement the concept have been within industrial practice for a number of years. The single item about which there had initially been a question was of the time required by practical computer hardware to perform the tomographic calculations. It was determined this should not be a feasibility problem because the speed of a 386-type personal computer was found to be adequate to do the calculations on a sampling basis, given appropriate algorithm and software properties. Further, special-purpose digital computer hardware exists that can do the required vector multiplications for the size of arrays contemplated here in tens of milliseconds when and if more rapid measurements are determined to be required. Software requirements were addressed only to the extent that they entered into these time estimates.

From an electronic-systems viewpoint, the concept is likewise feasible. 


\subsection{Discussion and Conclusions}

Essentially all food materials have structural properties analogous to those of potatoes. It is therefore reasonable to expect their acoustic properties to behave qualitatively in the same way, and to consider any differences as a matter of calibration, at most. On this basis, the properties of food materials present no obstacle to concept feasibility.

The use of tomography to locate and determine the temperature of particulates in the process stream is the key to the concept, potentially solving many longstanding problems with the application of ultrasound to foodprocessing. The present effort has shown that spatial resolution, sensitivity, and accuracy that are adequate for the purpose should be achievable within practical constraints. This same effort has developed design parameters and limits that will guide actual designs.

A conceptual study has shown that a sensor design meeting tomographic requirements should also be feasible, and its fabrication practicable. Furthermore, there is no question that electronic and computer systems having the requisite properties are readily available.

In summary, the study has uncovered no technical barriers to feasibility.

As with any new concept, there will be difficult engineering problems to be solved in each of the four technical areas. The largest of these is in tomography. A number of individually small effects, such as those of refraction and diffraction, were neglected in the calculations in order to make the calculations manageable. As a result, the design rules developed in this study can be only guides; considerably more detail work needs to be conducted to obtain a final design. The situation is similar for the sensor design. Finally, the acoustic measurements showed that food materials have, qualitatively, the properties appropriate to particulate-internal temperature measurements, but significant quantitative details need to be elucidated before a commercial unit becomes practicable. 
It is expected that other unknowns will also arise as work proceeds. The examples given are engineering problems, not feasibility questions, and are best resolved by actualily building and testing a laboratory-scale prototype device. The design of a practical Sonic Sensor System will require a number of intricate tradeoffs among each of these areas, a closely coordinated design effort that must be based on solid measurements using a prototype.

The question of economic feasibility was addressed in this study only indirectly because of limited ability at this time to project costs for a device whose final conceptual design has not been performed and for which performance requirements have only been defined indirectly. However, initial calculations suggest that the follow-on designs based on the laboratory prototype device for measuring particulates in cans would have the attractive simple payback period of a year or less. (See Appendix A). 


\section{PROJECTIONS}

\subsection{Plans for Phase II Work}

A laboratory prototype sensor is the next logical step in developing this concept. Such a device would provide a capability for noninvasive measurement of particulate temperature within sealed cans, and could readily be used on the output of retorts without wastage of samples that attends conventional practice.

The device would have two basic purposes: (a) to gain an engineering experience base by designing, building, and testing a complete sensor system, and (b) to obtain quantitative data on real food particulates under commercial processing conditions, especially variations in processing parameters and food-material properties.

The design of this prototype should provide for wide variations of sensor, data acquisition, and tomographic parameters in order to allow acquisition of data during the testing phase for fine-tuning of follow-on designs.

The testing cycle should emphasize calibration and parameter optimization for an expanded variety of food products under realistic processing conditions in a two-stage test program. The first stage would involve testing, data acquisition, and optimization, conducted at the NFPA pilot plant; the second should prove the system capabilities at one or more commercial food processing plants during normal operations, using and fine-tuning optimized parameters.

The prototype is, by definition, intended as an intermediate step between concept feasibility demonstration and full industrial application in such areas as process control in conventional canning and in aseptic packaging. The advantages of this approach are readily seen and their value appreciated: 
- Food material issues are separated from design issues and from processing technology issues, so that each can be addressed separately

- Prototype operation and testing as described provides for easy data acquisition to optimize ultrasonic design, tomographic reconstruction, and data processing and presentation. These latter issues will be addressed in conjunction with personnel and organizations who will actually be using any follow-on commercial units

- Calibrations can be obtained under "real-world" environments of food materials, particulate sizes, and processing conditions

- Real plant experience can be expected to enhance the usefulness and reliability of future designs

- The feasibility and utility of the concept is demonstrated conclusively to the food processing industry during the testing phase, enhancing the probability of market penetration by commercial devices.

The potential energy and economic savings achievable by this technology are addressed in Appendix $A$.

\subsection{Beyond Phase II}

Development and commercialization work beyond Phase II should pursue a two-pronged apprnach:

1. Pursue the development and marketing of a commercial instrument based on the successfully proven laboratory prototype for application in process control in conventional retort batch processing, replacing the present puncture-and-thermometer methods. Potential energy and cost savings, combined with potential 
improvement in product quality, and payback, should make this device attractive. The device is likely to be much simpler than the prototype because it would not need all of the flexibility of a development prototype, which is essentially a laboratory device.

2. Develop and pursue the marketing of a pilot-line prototype of a device for continuous aseptic processing. It is in this area that the largest energy and cost savings exist, and the potential for improvement of product quality is greatest. The key to this device is the development of a practical and cost-effective ultrasonic sensing head and electronic package; the engineering know-how for design of this package for a commercial unit should be available at the end of Phase II.

The NFPA and the food processing industry should be heavily involved in al1 aspects of both of these developments above, and should probably take the lead in initiating them, once Phase II demonstrations are successfully completed. The INEL participation should be limited primarily to the area of design of sensor head and electronic package combination. The design and performance requirements in this area are sufficiently unique that practical solutions to them are the subject of one or more current patent disclosures, and the capability to perform this design is not known to exist in practice in the food-processing equipment industry; other aspects of the designs are within the range of standard engineering practice in industry, once the design parameters are developed in Phase II.

FDA concurrence and regulatory changes will probably be needed for the use of either device. The NFPA should be aggressive in bringing FDA on board as eariy as possible, so that FDA concerns can be addressed in design and testing phases.

Food-freezing technology is the second of several areas where this technology is applicable, and where there is the potential for significant energy savings. The opportunity to explore these additional applications should be taken in this phase of the development. 


\section{References}

1. C. Javanaud, "Applifations of U1trasound to Food Systems, "U1trasonics 26, May 1988 , p. 117

2. Gabor T. Herman, "Image Reconstruction from Projections, "Academic Press, 1980 , p. 4. 
APPENDIX A

Calculations

A-1 


\section{APPENDIX A}

\section{Calculations}

\section{Energy Savings}

- Food processing by retorting methods requires about $350 \mathrm{Btu} / \mathrm{lb}$.

- Aseptic processing requites 25 to $50 \mathrm{Btu} / \mathrm{lb}$

- The USA annually processes about 15 milition tons of food products by conventional canning processes.

Assuming $30 \mathrm{Btu} / \mathrm{Ib}$ and $100 \%$ market penetration, aseptic processing would save

$(350-30) \times 15 \times 10^{6} \times 2000=9.6 \times 10^{12}=0.0096 \mathrm{quad} / \mathrm{y}$

without considering energy savings from better product utilization, less wastage, and significantly less energy for aseptic packaging, easily bringing the total to more than 0.01 quad.

2. Economic payback for non-invasive temperature-measuring device used on output of conventional (retort) process:

- A large processing plant will puncture (and waste) $10^{5} \mathrm{cans} / \mathrm{y}$ for temperature measurement during its season.

- Typical investment in can and contents is $\$ 0.50$ cost to the processor $=\$ 50 \mathrm{~K}$ plus disposal costs, which may be estimated at -\$20K in such states as Callfornia.

- The ultrasonic device is estimated to cost no more than $\$ 50 \mathrm{~K}$ to build in serial production, based on a 386-type personal computer. 
Simple-payback perlod would thus be a year or less, potentially' making the device financlally attractive. 

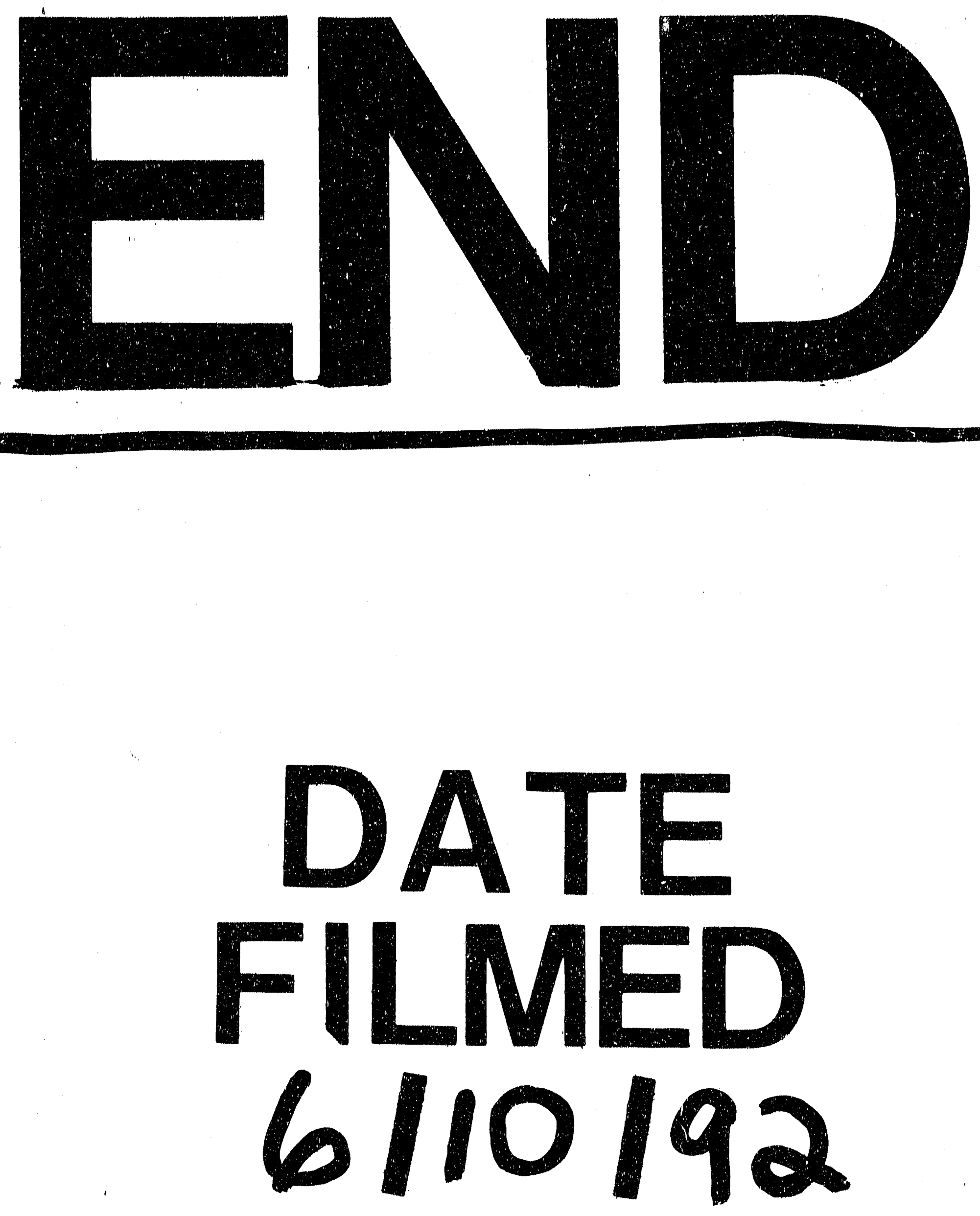


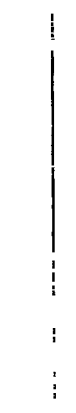

;

, 